\title{
HIGHER DISEASE DAMAGE IN PATIENTS WITH LATE-ONSET SYSTEMIC LUPUS ERYTHEMATOSUS
}

Gabriellen Vitiello Teixeira 1", , José Pinto de Souza Neto', Anna Beatriz Gomes Souza Duarte", Elisa Guimarães Motta', Marilena Mesquita Leal Fernandes ${ }^{1}$, Mauro Goldfarb', Adriana Danowski'

1. Hospital Federal dos Servidores do Estado, Rio de Janeiro (RJ), Brazil.

*Corresponding author: gabriellenvitiello@gmail.com

\section{BACKGROUND}

The objectives of this study were to compare disease characteristics and outcomes of late-onset systemic lupus erythematosus (SLE) and adult onset SLE.

\section{MATERIALS AND METHODS}

A cross-sectional study was performed in patients followed longitudinally in SLE cohort, accordingly to a standard protocol and collected by a computerized database. Late-onset was defined as diagnosed $\geq 50$ years of age and adult-onset between 18 and 49 years. The clinical features, serology, complications and the damage index Systemic Lupus International Collaborating Clinics / American College of Rheumatology Damage Index (SLICC / ACR SDI) were compared between the two groups of patients. Univariate analyses were carried out on each potential predictive factor with Pearson's correlation or the Fisher's test when appropriate.

\section{RESULTS}

Six hundred and eight patients were selected, 94.1\% female, 40.8\% Caucasian. After excluding the patients diagnosed before 18 years old, our total was 536 patients. Sixty-four (11.9\%) patients were diagnosed $\geq 50$ years of age. Patients with late-onset SLE showed a higher risk of developing damage accrual ( $p=0.02$; odds ratio, $\mathrm{OR}=2.03 ; 95 \% \mathrm{Cl}=1.07-3.84$ ), diabetes mellitus (DM) $(p<0.01 ; O R=5.25 ; 95 \% \mathrm{Cl}=2.65-10.39)$, acute myocardial infarction $(\mathrm{MI})(\mathrm{p}=0.01 ; \mathrm{OR}=3.34 ; 95 \% \mathrm{Cl}=1.23-0.84)$, osteoporosis $(p=0.00 ; O R=2.75 ; 95 \% C l=1.47-5.12)$, cataracts $(p=0.00 ; O R=2.35 ; 95 \% \mathrm{Cl}=1.23-9.05)$ and systemic arterial hypertension $(\mathrm{SAH})(p=0.02 ; \mathrm{OR}=2.48 ; 95 \% \mathrm{Cl}=1.38-4.45)$. Malar rash $(\mathrm{p}=0.01 ; \mathrm{OR}=0.50 ; 95 \% \mathrm{Cl}=0.29-0.85)$ was the single clinical manifestation with a lower chance of being found in patients with late diagnosis. The presence of anti-RNP $(p=0.02 ; O R=0.49$; $95 \% \mathrm{Cl}=0.25-0.93)$ was less frequent in those patients with a late diagnosis. There was no other significant difference between the populations.

\section{CONCLUSIONS}

Patients with late-onset SLE presented more cardiovascular complications, such as DM, MI and SAH, when compared to patients with early diagnosis of the disease. They also have more complications such as cataracts and osteoporosis, and, consequently, higher damage accrual. These results may be associated with cardiovascular risk and aging. We could not establish major differences in the clinical picture or the serology of these patients. Therefore, co-morbidities and damage prevention should be major targets when treating late-onset SLE. 